\title{
Mobility Management in a Plug and Play Architecture
}

\author{
Mazen Malek and Finn Arve Aagesen \\ Department of Telematics, Norwegian University of Science and Technology, Trondheim, \\ Norway
}

malek@item.ntnu.no,aagesen@item.ntnu.no

\begin{abstract}
Mobility Management functionality in the Plug-and-Play ( $\mathrm{PaP})$ architecture is presented comprising different approaches for Actor, Terminal, User and Session Mobility Management. We explore a few issues related to implementation design and propose a set of components to facilitate the deployment of this platform in the available $\mathrm{PaP}$ applications. The $\mathrm{PaP}$ architecture is briefly introduced and subsequent definitions and terms are dealt with. The architecture itself is based on a theatre metaphor, in which plays define the functionality of the system. PaP components are realised by actors playing roles defined by manuscripts. An actor's capabilities define his possibilities for playing various roles. The mobility management is introduced to add a capability to handle any move of any constituent of the system. This could range from moving functionality, or actors, to moving users of the system. Throughout the paper we try to give a survey on the various mobility cases and make an effort to demonstrate an early set of mobility management algorithms or methods.
\end{abstract}

Keywords: Plug and Play, mobility management, teleservice architecture, active networks.

\section{INTRODUCTION}

Grade of network intelligence is defined as the efficient flexibility in the introduction of new teleservices and the efficient flexibility in the execution of teleservices. IN (Intelligent Networks) [ITU92], TINA (Telecommunication Information Networking Architecture) [TINA95], 
Mobile Agents and Active Networks ([Bies97], [Bies98], [Raza99], [Tenn97]) are all solutions aimed to improve the network intelligence.

Plug-and-play $(\mathrm{PaP})$ for telecommunications means that the hardware and software parts have the ability to configure themselves when installed into a network and then to provide services according to their own capabilities, the service repertoire and the operating policies of the system. Plug-and-play functionality means utterly increase of network intelligence. The concept $\mathrm{PaP}$ stems from the personal computing area. $\mathrm{PaP}$ simply means that you plug-in and then the system works. In these systems, the plugged in component as well as the framework has a predefined functionality. We denote this static $\mathrm{PaP}$. A more general kind of $\mathrm{PaP}$ is when the plugged-in unit has a set of basic capabilities, but its functionality is defined as a part of the plug-in procedure and it can be changed dynamically. We denote this as dynamic PaP. An example is a cellular phone, which obtains the services it provides depending on its inherent capabilities, which user that logs on, and which network it is attached to. The focus of this paper is on dynamic PaP, and from now on PaP means dynamic $\mathrm{PaP}$. For a detailed description of the $\mathrm{PaP}$ architecture and the ongoing research activities around this project please refer to: (http://www.item.ntnu.no/ plugandplay).

For purposes of more flexible computational capabilities, utilisation and adaptation of wider range of network services and possible introduction of network management tasks, our PaP model should be enhanced with various mobility management schemes. This comprises Actor Mobility, Terminal Mobility, User and Session Mobility.

The very basic requirement in this regard is the ability to move already instantiated functionality among nodes or components. In this paper we refer to this by Actor Mobility. Actor is one of the core concepts of the $\mathrm{PaP}$ architecture. An Actor constitutes a Role-Figure by behaving according to a defined role. An Actor has the capability to play different roles and then to constitute various Role-Figures. An Actor can make up different roles during its lifetime. Movement of functionality to the generic Actor by the time when the Role-Figure is instantiated or changed is already a core mechanism of the PaP architecture. Referring to the concept model in Figure 1, the Actor Mobility is the physical movement of Role Figures.

Now what is the difference between this $\mathrm{PaP}$ architecture, Active networks and Mobile Agents [Bies97]. The PaP architecture is based on Role-Figures, which have the power of EFSM (Extended Finite State Machines). All network and teleservice behaviour are assumed to be based on the same $\mathrm{PaP}$ architecture, in which Role-Figures get their behaviour based on Code-on-Demand. Actor mobility in this architecture is different from Active Networks and Mobile Agents in the sense that movement conserves state and relationships to already ongoing sessions with other Role-Figures. The picture is not the almost independent piece of program that travels around with licence to do more or less independent actions. The picture is, instead, the movement of a program with well-defined behaviour, 
and which already has a lot of bindings that need to be conserved. In our case the actors are just a representation of the functionality at a given node. When actors move the execution of the functionality is moved to a different node. Section 3 provides a clearer view of Actor Mobility.

Nowadays telecommunication networks provide the users with ever improving and flexible mobility in both wired and wireless technologies. Users could move their terminals more and more freely, and yet be able to access the same range of services. This ability adds a great challenge in terms of managing terminal mobility. Terminal Mobility is handled in Section 4. For the remaining types of mobility, the User and Session mobility, sections 5 and 6 give an overview of the proposed methods. Finally we give summary and conclusions that include our further interests and future work.

\section{A PAP REFERENCE ARCHITECTURE}

In this section we give a brief description of the $\mathrm{PaP}$ architecture, how does it define functionality and what are its basic constituents. The PaP was introduced in [Aage99]. Important definitions are highlighted and the basic procedures are mentioned. Some figures illustrating the different object models and view points are also included to add clarity.

\subsection{PaP functional object model}

PaP components: are real-world active hardware and/or software modules. These can be combined hardware/software modules with one or more external hardware interfaces, or pure software modules. These must interface with a software platform capable of running $\mathrm{PaP}$ application software. $\mathrm{PaP}$ components are composed from (one or more) interacting instances of $\mathrm{PaP}$ functional objects, where each instance is defined by reference to an object type. This means that the $\mathrm{PaP}$ component functionality is defined by a functional object model consisting of functional $\mathrm{PaP}$ objects. ISO's reference model for Open Distributed Processing (ODP) [Duts96] defines the enterprise, computational, information, engineering and technical viewpoints. The viewpoints of primary interest with respect to $\mathrm{PaP}$ are the computational and the engineering viewpoints. The $\mathrm{PaP}$ components are basically engineering viewpoint objects. The relationship between important PaP concepts is shown in Figure 1.

PaP Actor: An actor is a generic object with a generic behaviour. Actors are able to behave according to a manuscript. The repertoire consists of plays, which are defined by roles and the role is formalised by a manuscript. These concepts have meaning similar to as those that are used in the theatre context. The manuscript is the functional $\mathrm{PaP}$ object type definition. An instance of a PaP functional object, also here denoted as a role-figure is realised by an actor, which is executing the manuscript. 
PaP Manuscript: defines the entire behaviour of an actor. Rolesession is a projection of the behaviour of the actor with respect to one of its interacting actors. The entire role as well as the role-sessions are EFSMs (Extended Finite State Machines). An actor also has a defined set of capabilities, which is the ability or power to do something. The capabilities are the result of the available hardware functionality connected to the hardware executing the actor software behaviour, but also the quantitative aspects such as processing capacity [Aage99].

PaP Play: is a defined autonomous functionality. The play defines the context for relationships between $\mathrm{PaP}$ objects as well as their behaviour. One important $\mathrm{PaP}$ object instance necessary to initialise any play is the director role-function. Director behaviour is also defined by an instance of a play. An actor has three distinct behaviour phases: 1) the plug-in phase, 2) the play phase and 3) the plug-out phase.

PaP support functionality: The following functions are needed: PlayPlugIn, PlayChangesPlugIn, PlayPlugOut, ActorPlugIn, ActorPlugOut, ActorBehaviourPlugIn, ActorPlay, RoleSessionAction, Subscribe, ActorChangeBehaviour, ActorBehaviourPlugOut and ChangeActorCapabilities. For details see ([Aage99], [Joha99]). The functions: ActorBehaviourPlugIn, ActorPlay and ActorBehaviourPlugOut comprise the initialisation of a generic actor pending for a manuscript, performing the manuscript, and finally making the actor pending for a new manuscript. This functionality with the addition of ActorChangeBehaviour is denoted as the basic PaP functionality. The actor is initialised by first activating its director. An actor negotiates with a director role-figure in order to obtain its behaviour. The director role-figure will create an instance of a manuscript with all necessary parameters bound particularly for the actor. The director role-figure also acts as a binding object, which helps to establish communication or interactions among actors. After receiving a manuscript from the director role-figure, an actor will start acting according to the specification described in the manuscript. From this point on in time, the actor becomes autonomous and independent of the director role-figure and constitutes an application role-figure until it terminates or wants to change its behaviour. 


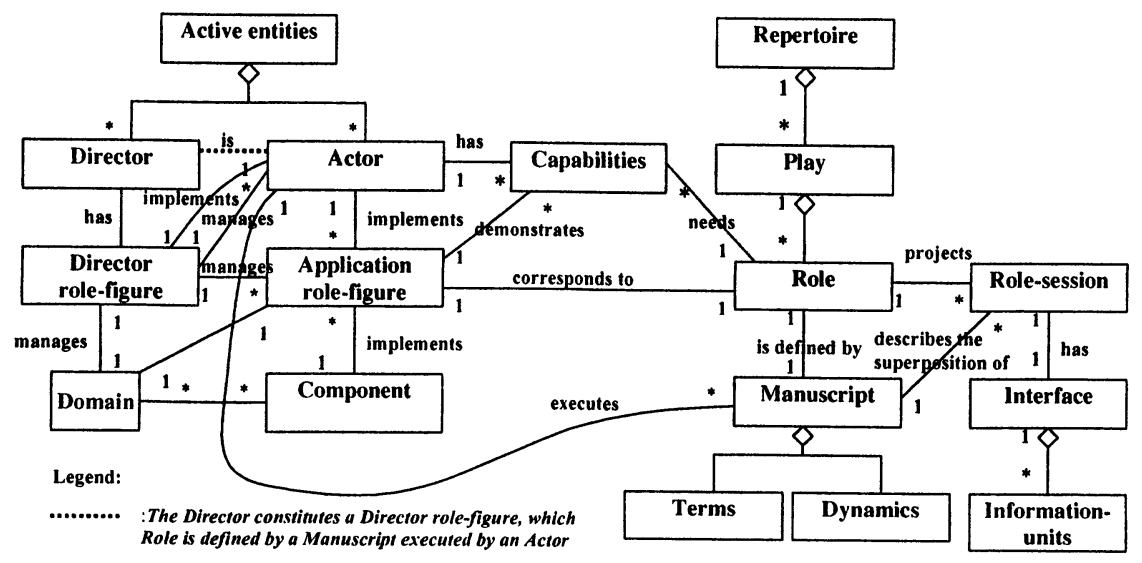

Figure 1. PaP concepts

\subsection{PaP Engineering Model}

As illustrated in figure 1, there are two types of role-figures, the application role-figure and the director role-figure. A relation between an application role-figure and a director role-figure must always exist. Relations between director role-figures will give a possibility to obtain a distributed solution for the director role. A PaP system with more than one director needs administrative domains to manage the federation of responsibility between director role-figures. Figure 2 shows the structuring of the needed functionality into five layers. All layers in except for $P a P$ specific applications and non-PaP applications are completely independent of the applications themselves. The PaP functionality will have to interface to some infrastructure technology at the bottom layer, and may interface with any type of non $\mathrm{PaP}$ application through the top layer. 


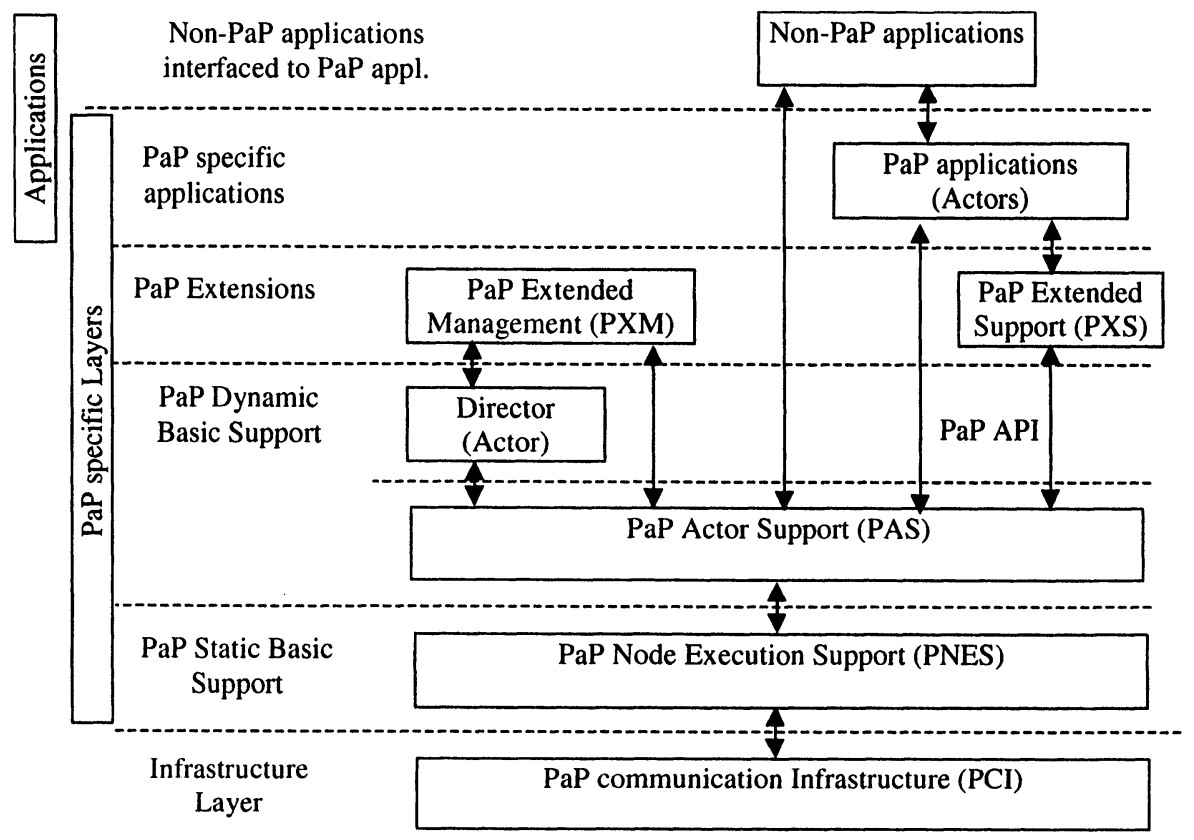

Figure 2. PaP layered model

A PaP communication infrastructure $(P C I)$ architecture based on standard solutions, will usually consist of three layers with the operating system functionality (e.g. Unix or Windows) at the bottom, the network communication functionality (e.g. TCP/IP) in the middle, and some distributed system solution (e.g. CORBA ORB or Java RMI) at the top. The PCI top layer may be omitted, but that will require a more complex implementation of the interfacing module PNES if the PaP functionality require a distributed system solution.

PaP Node Execution Support (PNES) makes it possible to run PaP software on a node, and for $\mathrm{PaP}$ functionality (i.e. executed by actors) on different nodes to interact with each other. PNES is able to receive requests from other PNESes, interpret these requests and take proper actions. PNES will also do start-up and initialisation of PASes or PCIs if that is required. PNES implements the PaP functionality that is termed the PaP Static Basic Support in the model. Static in this sense means that changes/extensions of the PNES functionality must be backward compatible with earlier versions because this functionality represents the "bootstrap" that is necessary to be able to run $\mathrm{PaP}$ applications. Only this functionality must be manually installed at a node before $\mathrm{PaP}$ applications can be installed and activated.

PaP Actor Support (PAS) makes it possible to create actors within the context of an operating system process/thread, to give these actors behaviour, and to communicate information between these actors and their environments. 
Director is both responsible for the management of the $\mathrm{PaP}$ application definitions, i.e. its part of the repertoire- and manuscript-bases, and for the management of information concerning actors, i.e. its playingbase. A director is involved in many of the functions related to the services provided by PAS.

\section{ACTOR MOBILITY}

Actor mobility here stands for the movement of instantiated functionality at a node, or the Role-Figure executed by an actor. Actor mobility could be achieved by extending actors capabilities with movement ability, and by keeping track of this movement. In other words, the actor location specific information should be updated whenever an actor moves. In the $\mathrm{PaP}$ terminology we use Global Actor Identifier (GAI) as an object that maintains actor specific information regarding which PNES and PAS instances the actor executes in. Definitely, the association between actors, directors, actor-actor relations and capabilities should be dealt with carefully.

Actors move due to requirements on resource limitation, configuration change, functionality change, etc. In order to support actor mobility, the system should be able to deactivate and reactivate moving actors transparently. Therefore moved actors should be able to continue their behaviour from the point when they started to move. For this we need to define the concept of actor state that defines actor's state of a play. This aims at defining different phases of actor's behaviour and consequently the movement process will be reduced into an extended request of ActorPlugIn. As far as the implementation design is concerned, actor manuscripts should define actor's behaviour and interactions with other actors using state-based objects. On the other hand, the movement (transition) of actors should be handled using a proper method. In the following we will introduce three method case studies that are based on replicating moved actors, using proxy to forward requests and applying a centralised agent. But before that we need to figure out what are the possibilities of actor mobility.

When actors need to move from PAS to PAS or from node to node (a node is equivalent to a PNES instance in the PaP terminology), full update of actor specific information should be applied at all instances that are related to this actor. Whenever an actor is activated it is associated to a PNES, PAS and a home director instances. PNES holds the GAI that is needed to route requests coming to this actor. PAS is the application (process or thread) in which actor objects are executing. Directors are related to actors through the definition of actors "home interface". This definition is crucial for directors to keep track of actors' location. Actor movement could be classified into three different scenarios: 
1. Actor movement from PAS to PAS - in the same PNES instance

2. Actor movement from PNES to PNES - having the same director

3. Actor movement from PNES to PNES - having different directors

\subsection{Actor Mobility Management methods}

AMM method 1: The first method to be utilized is based on employing a single copy of a moved actor at the new location and keeping all information bases as they are. All active role sessions of that actor should be moved to the new location. Routing of requests will be done as if there was no movement at all, except for that the old actor will forward all arriving requests to the new location. This method is restricted for certain types of movement scenarios, namely 1 and 2 as described in the previous section. The transition period seems to be short and simple, however further movements of the same actor should be carried out with care. Thus instead of forwarding move requests to the new location, an ActorPlugOut request and a new ActorPlugIn request should be initiated. Figure 3 illustrate a case where an actor moves to a new location, and then moves to another location. In this figure we apply a general type of request to the actors and denote them by request 1 and request 2 for simplicity. Also for the other requests, e.g. ActorPlugIn and ActorMove, we don't use parameters, as well as we avoid detailed interactions with directors.

AMM Method 2: This is a more general method that allows actors to move freely and change "home interface" or associated director. Actors as objects will be created at different locations and will not be kept at their original locations. A full update of the system is required here, in which the actor identity should remain the same except for its location that need to be changed. This method is more flexible from the performance point of view, but more complex in the implementation. An updated actor location will be always available at the entity registries of the middlewere platform, such as RMI technology. Figure 4 demonstrates how this method works. Note that request 2 is directed to the new location by the request sender.

AMM Method 3: This method is based on centralised agent that acts as a mobility manager within the context of $\mathrm{PaP}$ architecture, as in figure 5. The whole PaP functionality should be modified in a way that it could include a mobility service that actors would broadcast to or consult before they move or send requests respectively. "LocationUpdate" procedure should be used prior to movements and "ActorDiscovery" procedure should be used prior to request routing. This method would be the most flexible and robust to comply with movements of actors. 


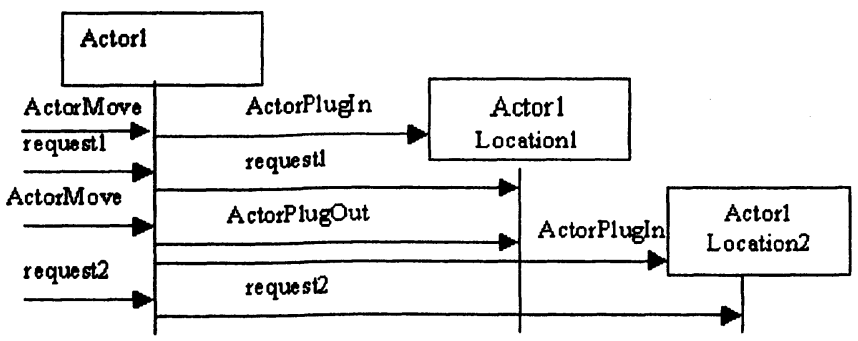

Figure 3. AMM method 1.

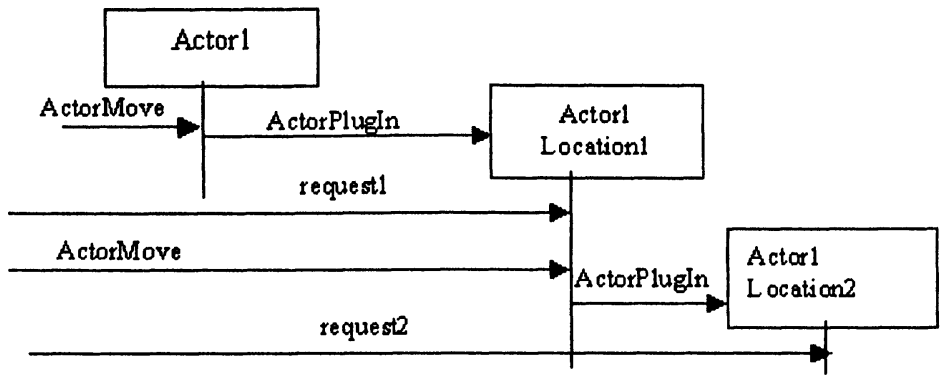

Figure 4. AMM method 2

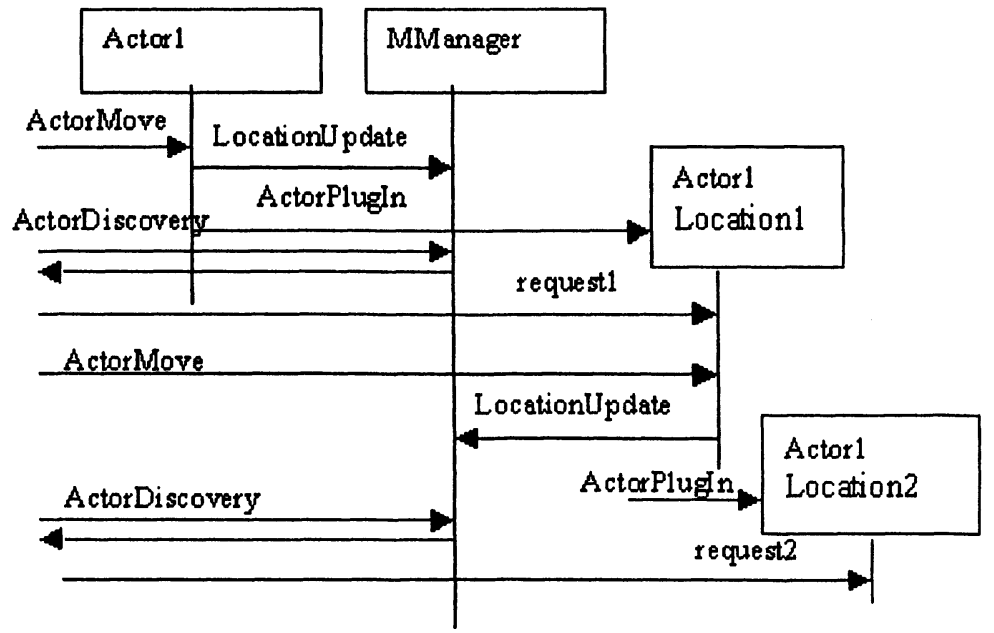

Figure 5. AMM method 3 


\section{TERMINAL MOBILITY}

In the $\mathrm{PaP}$ architectural concept there is no special definition for terminals or nodes, instead $\mathrm{PaP}$ components are defined to be the real world active modules that take part in the $\mathrm{PaP}$ functionality. A terminal could be a network node that realizes the interface towards the end user. The mobility of a terminal or a node is defined by the change of its physical address. This is clearly seen in the engineering view of the PaP system, where at each node there is a PNES instance running at a distinct network address. To achieve mobility management for these moving nodes we need to keep track of their movements. So a manager should be responsible for updating the locations of all nodes that participate in a possible $\mathrm{PaP}$ application related to a movement. The knowledge of this central agent will be imbedded in the PNES objects, and whenever it moves all PNES objects are updated. This is better demonstrated by an example. Assume first a URL address as the initial access point where the PaP support software and application are available. Assume also a terminal, possibly a laptop, which would like to access this application. The first step is to download the PaP bootstrap and modify some configuration file stating the webserver from which the application will be downloaded and a default director interface, which used to be the localhost. Once this initial phase is ready the PNES could be started from which a play will be loaded and some actors could be plugged in to perform some role figures and interact with each other. Assume now the terminal will change location, e.g. connect to the network at different place using DHCP. Note that the terminal will not be switched off. The terminal will try to resume communicating according to the behaviour of its actors. On the other hand, the rest of the communicating nodes or terminals should be able to discover this move before, while and after it is being performed.

The proposed solution is based on mobility manager or MManager, which runs at an address known to all other nodes. MManager will run when the first PNES be started, and its network location should be part of the configuration file. Secondly, at each communicating node there should be a special agent performing location update and location query procedures. This agent could be called MAgent that stands for mobility agent, as in figure 6. MAgents are responsible for notifying MManager if there is a change in the node's address. On the other hand, they should send location query on every communication event with other nodes. The MManager responds to location updates by updating the node's location, and replies with the current location of the requested node upon receiving a location query. All in all, terminal mobility management is a kind of play that runs in the background of any $\mathrm{PaP}$ system that supports terminal mobility.

To obtain a clearer view of how nodes interact figure 7 shows a possible node-to-node interaction and their corresponding actors. This figure corresponds to moving node 1 in figure 6. The Actor-environment- 
execution-module (AEEM) is a process/thread that executes a collection of actors with associated PaP Actor Support (PAS).

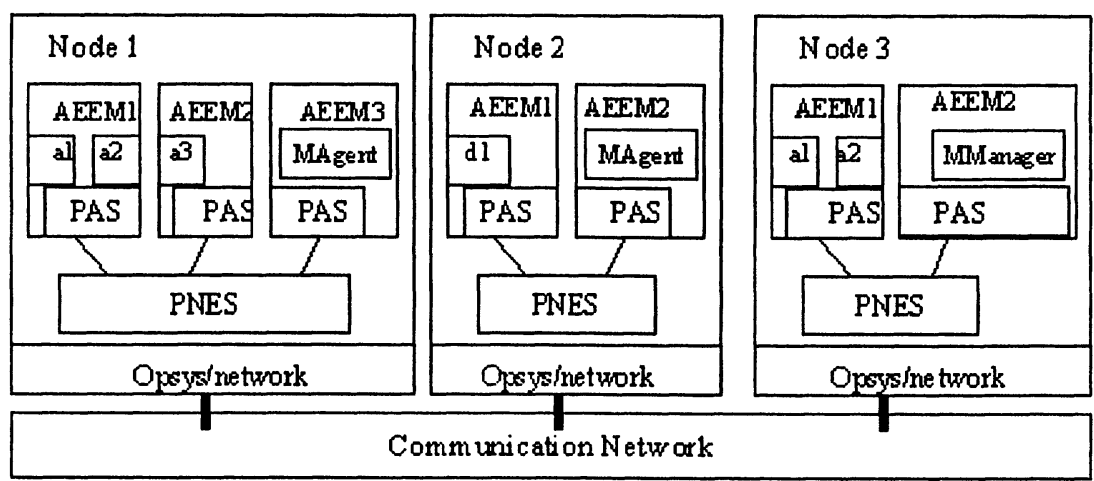

Figure 6. Engineering view of three $\mathrm{PaP}$ nodes with terminal mobility management.

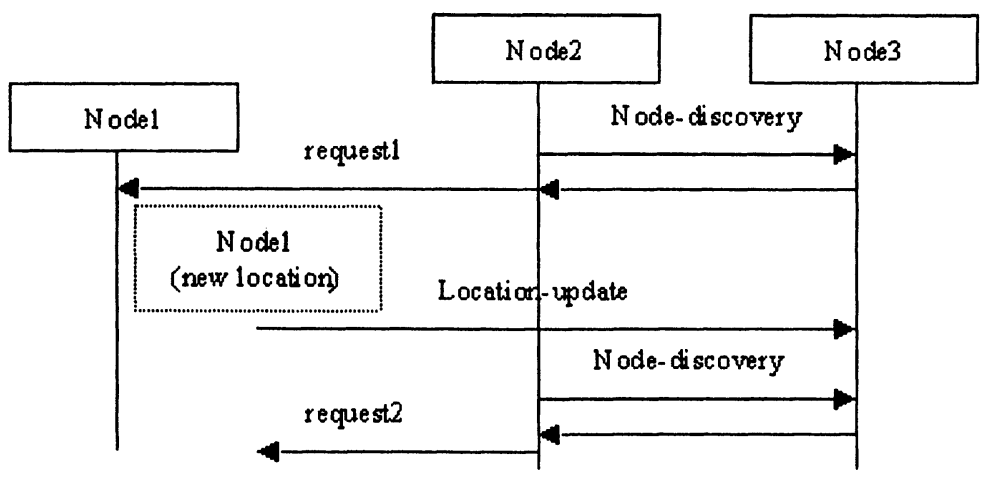

Figure 7. Node interaction before and after node moves

\section{USER MOBILITY}

This is also known as personal mobility, which is the utilization of services that are personalized with end users' preferences and identities independently of physical location and specific equipment. In this context we need to define the user and the services in a candidate $\mathrm{PaP}$ system. Services are carried out by service components, which are actors. In order to achieve user mobility we need to dedicate a special actor to behave as a provider agent [Axel98]. In our proposal we suggest to assign an agent to 
each user, which is an actor or a set of actors carrying out system interface towards the user. This will be called User Agent (UAgent).

When we discuss user mobility we are actually talking about users and their existence in a certain domain. So services that are utilizable for a user at certain domain are not necessarily available at another domain. If a user could access all services provided to him by his home domain through another domain then these domains support user mobility. In the following we suggest a solution to achieve this. When a user moves from his home domain into a visitor domain he will first access the $\mathrm{PaP}$ service provided by the visitor domain's director, or director 2 in figure 8 and 9. This service should be able to grant some users visitor status (a guest type access to basic functionality). After some initial interaction with this domain, this user will be offered to access his home domain. Meanwhile, the user is a user of the visitor domain, so he requires a UAgent. However, as this user chooses to access the services provided by his home domain then a Visitor Agent (VAgent) should be assigned to him that is controlled by the home director, director 1. VAgent has similar definition to UAgent.

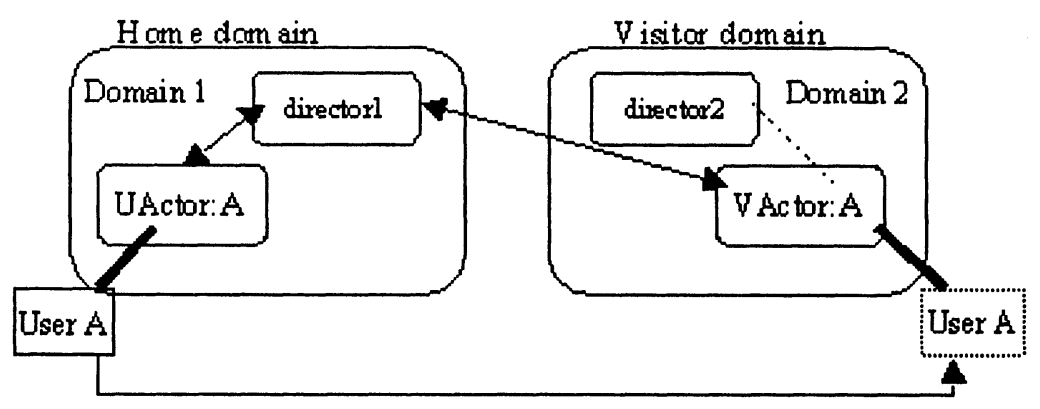

Figure 8. A proposal to handle user mobility

To realize personal mobility service, personalized environment is constructed according to the user profile of the end user. According to the service architecture of TINA-C this is defined as an aggregation of four informational objects: usage context, service profile, session description and user registration. The user profile object should be moved to the visited domain in order to construct the user's environment. In our architecture this issue is less complex as we associate the VAgent to the home domain, or home director. By this way, any needed objects, e.g. the user's profile, could be accessed and downloaded from within the VAgent without any interaction with the visited domain. The main issue here is, however, at the beginning of the registration process. We should note that these domains are not business administration domains, they are instances of executed or performed plays controlled by directors. 


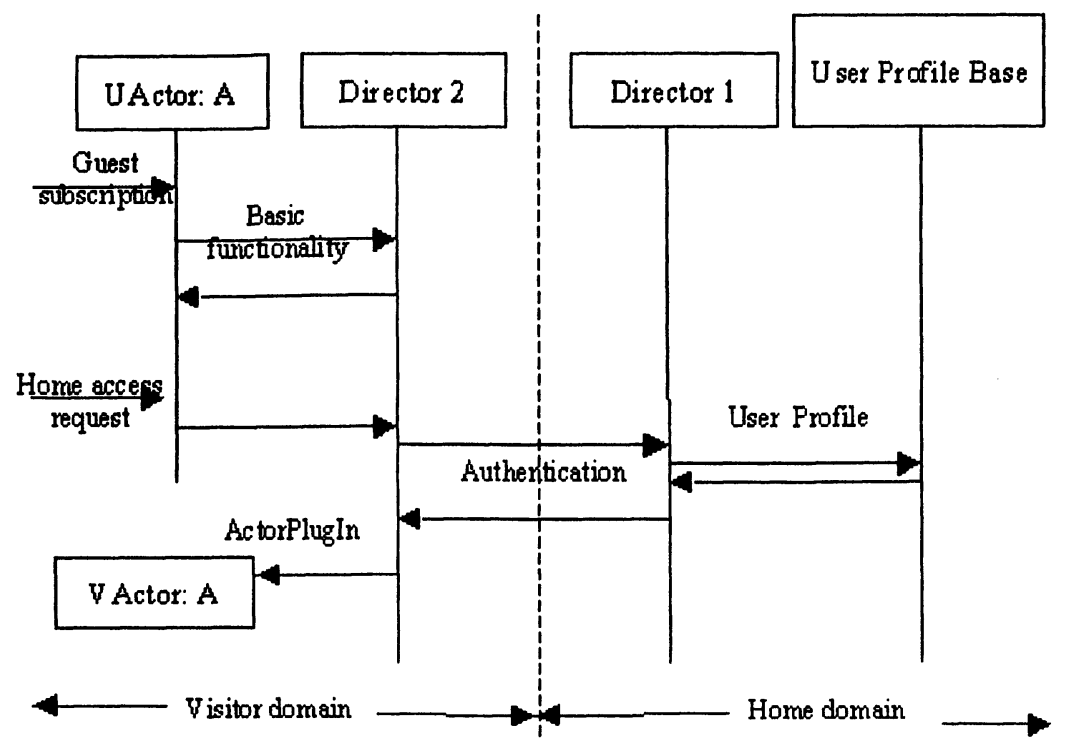

Figure 9. User mobility support: subscription, authentication and configuration.

\section{SESSION MOBILITY}

Session is an interaction collection who's goal is to satisfy the goal of a service by performing activities during a specific period of time. Session is also associated with the allocation of resources that are necessary to execute the aimed service. There are two types of sessions: User/Access and Service/Communication sessions.

Here we should recall the UAgent from the previous section and give it a broader definition: a computational object that manages user's preferences and performs all the operations and tasks required by the user. We should recall also that PNES object carries enough information about the node where the user is seeking access.

For the first type of session the user actor concept seems to be capable of providing this mobility. A user is granted an access to the system by assigning a UAgent. When it demands the same service from different domain a VAgent is assigned. To support the second type of session mobility we need to introduce a new role to the $\mathrm{PaP}$ architecture, this is the session manager.

We shall consider two requests to serve as a basis for session mobility: suspend and resume. A session is always initiated whenever a user is subscribed to the system and demands one of its services. The session manager is invoked and asked for a session ID. Now on, this session ID will be addressed at any future operation for session mobility. Since there is 
always a special actor associated with users behaviour, UAgent or VAgent, and since these actors behave according to some state transition scheme, then resuming of any suspended session is achieved by recapturing the actor's behaviour at the state where the session was stopped.

\section{SUMMARY AND CONCLUSIONS}

In this paper a mobility management functionality based on a $\mathrm{PaP}$ architecture was introduced. Support for Actor, Terminal, User and Session mobility is the main part of a platform realising this functionality. Some parts of the proposed platform are being integrated to the available demonstrator application, the tele-school application, which is based on Java RMI technology. The Actor mobility Management methods are the first parts to be integrated, as they don't require a major shift to the basic functionality. The AMM1 method in particular provides basic and yet powerful actor mobility to the $\mathrm{PaP}$ architecture. The idea is basically an actor proxy scheme for moving actors.

The paper dealt also with terminal mobility that is an introduction of mobile nodes. Some implementation specific details were elaborated, and various aspects of the modified architecture were mentioned. New requests and procedures will be needed to cope with this added capability. However, this addition is totally inline with the overall terminology, as we outline it as a $\mathrm{PaP}$ play (or service) running in the background of a $\mathrm{PaP}$ application. At last, we introduced a support for user and session mobility. This is mainly based on TINA definitions and its concept of a user and session. We believe our proposal for such mobility types benefits from the simplicity of mapping actors to directors in our architecture.

While in this article we laid out a platform for the integrated provision of different mobility types, there are several issues that need further investigation. On the one hand, we need to break up the overall $\mathrm{PaP}$ architecture into subsystems to provide selected types of mobility, which for some will be in the support and for others in the application layers. On the other hand, we need to find efficient ways to map our methods and procedures into proper applications and communicating platforms. We expect the approaches presented here to play an important role in bringing the plug and play concept to telecommunications in a more practical manner.

\section{REFERENCES}

[1] [ITU92] ITU-T, Principles of intelligent network architecture, October 1992.

[2] [TINA95] TINA Consortium, TINA-C Deliverable: Overall Concepts and Principles of TINA V1.0, February 1995. 
[3] [Bies97] Andrzej Bieszczad and Bernard Pagurek, Towards plug- and play networks with mobile code, proceedings of ICCC 97 , November 1997.

[4] [Bies98] Andrzej Bieszczad and Bernard Pagurek and Tony White, Mobile Agents for Network Management, IEEE Communications Surveys, volume 1 number 1, 1998.

[5] [Raza99] S. K. Raza and Andrzej Bieszczad, Network Configuration with Plug and Play Components, The Sixth IFIP/IEEE International Symposium on Integrated Network Management

[6] [Tenn97] David L. Tennenhouse, Jonathan M. Smith, David Sincoskie, David J. Wetherall and Gary J. Minden, A Survey of Active Network Research, IEEE Communications Magazine, Volume 35 no 1, 1997, pages 80-86.

[7] [Aage99] Finn Arve Aagesen, Bjarne E. Helvik, Vilas Wuwongse, Hein Meling, Rolv Braek and Ulrik Johansen, Towards A Plug and Play Architecture for Telecommunications, Proceedings of IFIP SMARTNET 99, Bangkok, November 1999.

[8] [Duts96] Joubine Dutszadeh and Elie Najm, Formal Support for ODP and Teleservices, Proceedings of the IFIP/ICCC conference on Information Network and Data Communication, June 1996.

[9] [Joha99] Ulrik Johansen, Finn Arve Aagesen, Bjarne E. Helvik and Hein Meling, Design Specification of the PaP Support Functionality, Plug-andPlay Technical Report, Department of Telematics, NTNU, 1999-12-10, ISSN 1500-3868

[10] [Axe198] Axel Kupper, User Agent - An approach for Service and User Management in $3^{\text {rd }}$ Generation Mobile Networks, International Conference on Telecommunications 1998. 